\title{
Was Germany’s Corona Lockdown Necessary?
}

Christof Kuhbandner ${ }^{1 * \mathbb{\pi}, \text { Stefan Homburg }}{ }^{2 \pi}$, Harald Walach ${ }^{3,4}$, Stefan Hockertz ${ }^{5}$

${ }^{1}$ University of Regensburg, Department of Human Sciences, Germany

${ }^{2}$ Leibniz University Hannover, Department of Public Finance, Germany

${ }^{3}$ Poznan University of Medical Sciences, Department of Pediatric Gastroenterology, Poznan,

Poland

${ }^{4}$ University of Witten-Herdecke, Department of Psychology, Witten, Germany

${ }^{5} \mathrm{CEO}$ tpi consult Gmbh, formerly Director of the Institute of Experimental and Clinical

Toxicology, University Hamburg.

*Correspondence to: christof.kuhbandner@ur.de

IThese authors contributed equally to this work. 


\begin{abstract}
During the spread of SARS-Cov-2, Germany imposed various restrictions, including an extensive lockdown on 23 March 2020. In this paper, we point out that the evaluation of the success of such policies depends critically on data quality. Using reported confirmed cases is apt to produce misleading results because these data come with unknown variable lags. Using data on incident cases, i.e., dates of the onset of symptoms, produces results that are much more reliable.
\end{abstract}

\title{
Introduction
}

During the recent SARS-Cov-2 pandemic, many countries adopted various nonpharmaceutical interventions (NPIs). These harmed the economies tremendously and had strong negative impacts on physical, mental, and social health conditions, such as increases in suicides or domestic violence [1-4]. Given such adverse effects, it is important to determine whether the measures were actually successful in curbing the spread of the coronavirus.

Germany prohibited large public gatherings on 9 March, closed its schools and other educational institutions on 16 March, and imposed an extensive lockdown and contact bans on 23 March. Were these interventions necessary to avoid a medical disaster? Some studies, such as Dehning et al. [5], use data on reported confirmed cases to evaluate the impacts of the various NPIs. Section 2 reviews their principal finding and points out serious problems with the data used. In section 3, we present an alternative approach that relies on official data provided by Germany's federal health agency, Robert Koch Institute (RKI). Section 4 concludes the paper.

\section{Data on Reported Cases}


Dehning et al. model the growth rate of SARS-CoV-2 infections in Germany using a Susceptible-Infected-Recovered (SIR) model combined with Bayesian parameter inference. The authors report change points in the growth rate that correspond closely to three NPIs that became effective on 9 March (prohibition of large public gatherings), 16 March (closing of schools and other educational institutions along with the closing of nonessential stores), and 23 March (extensive lockdown, including a contact ban). Their main conclusion (p. 4) reads "that the full extent of interventions was necessary to stop exponential growth". Fig 1 illustrates this central finding. It shows that the coronavirus grew with a positive effective growth rate until the first NPI, which slowed down the spread. The second NPI reduced the effective rate further, and the third one, the extensive lockdown, drove it into negative territory.

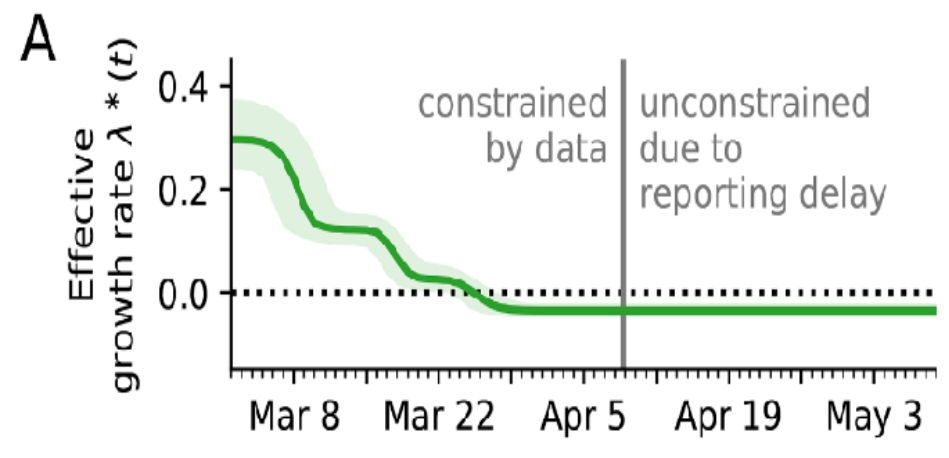

Fig 1. Effective growth rate of infections in Germany. Source: [5], Fig. 3A.

There are several fundamental methodological issues that cast serious doubt on the conclusions drawn by Dehning et al. Accounting for these issues suggests that the opposite of their principal inference is actually correct: neither of the governmental interventions could have had any effect on the spread of the virus because the number of new infections declined much earlier than estimated in their study. Furthermore, the authors ignore direct empirical evidence 
showing that such countermeasures had very low or even no effects. We consider their study to be seriously flawed.

To assess the potential effects of NPIs on the spread of a virus, it is crucial to determine the date of infection as exactly as possible. With misspecified infection dates, any conclusions about the effect of NPIs are meaningless. The authors estimated the date of infection based on the date when a confirmed case was reported, according to the Johns Hopkins University Center for Systems Science and Engineering (JHU CSSE) dashboard. To infer the infection date from the reporting date, they included a parameter in their SIR model that aims at determining the socalled 'reporting delay', i.e., the delay between infection date and reporting date. Critically, their parameter estimate is constrained by an informative prior that, in turn, is based on the assumption of an incubation period of 5-6 days and a test delay. Using their priors, the authors estimated a total delay of 8.6 days during the initial phase and 11.4 days during the later phase.

This procedure is inadequate. First, Dehning et al. use data from the JHU CSSE dashboard. As the Robert Koch Institute (RKI), Germany's federal health agency, points out in its profound FAQ section on the coronavirus [6], data from the JHU CSSE dashboard allow only limited conclusions because they stem from internet media reports and social media, and vary in reporting guidelines. Second, inferring infection dates from reporting dates would only make sense if reporting dates varied systematically with infection dates. However, the intervals between dates of actual infections, diagnostic testing, and reporting differ vastly across people. Many suspected people were tested even before symptom onset, whereas true patients were at times tested more than 20 days after symptom onset [7]. Therefore, it is hardly possible to conclude anything meaningful from modeling the spread of infections using reporting dates.

\section{Data on Incident Cases}


Germany's RKI, [8], published 15 April, employs a more sophisticated approach. Their model is not based on reporting dates but on identified dates of symptom onset, referred to as incident cases. With an established incubation period of 5 days (e.g. [9], 5.1 days, CI 95\% 4.5 to 5.8 days), incident cases reflect infection dates much more accurately. To describe the dynamics, RKI uses a growth factor R (reproduction number), which compares the 4-day mean of incident cases on one day with the corresponding mean 4 days before. By construction, $\mathrm{R}$ lags behind the actual dynamics by 4 days. To make our argument more succinct, we neglect this lag, consideration of which would strengthen our point. Fig 2 shows the actual growth factor of incident cases, R, determined by RKI.

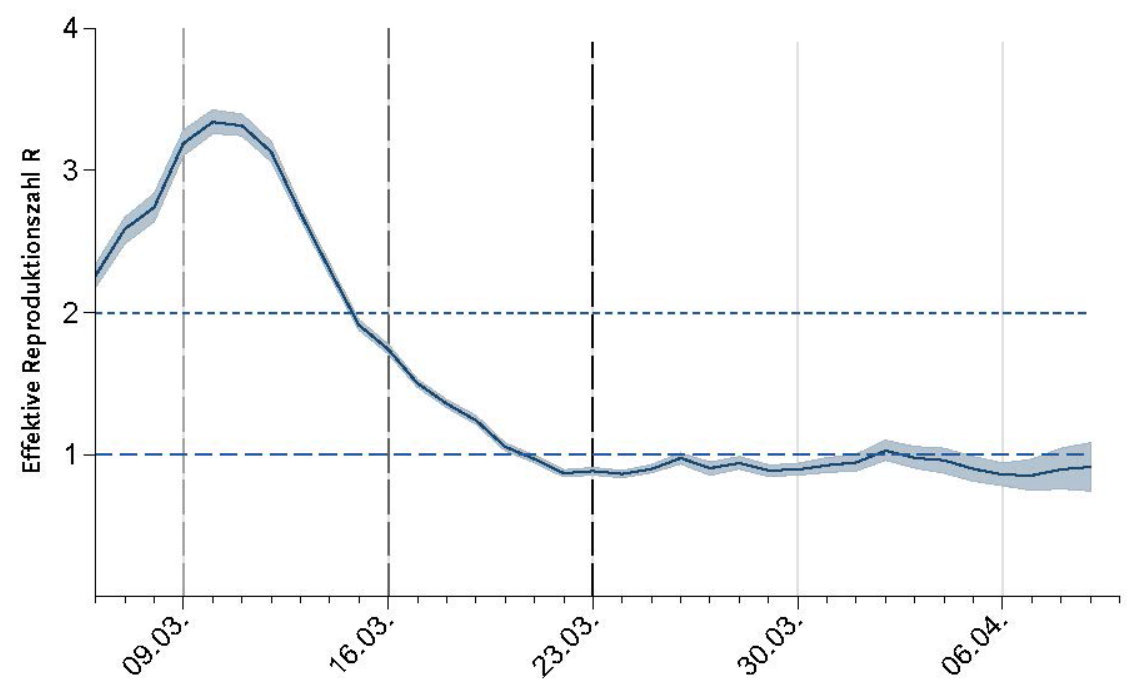

Fig 2. Growth factor of incident cases in Germany. Source: [8], Fig. 4.

Fig 2 documents that the growth of incident cases reached its maximum already on 10 March. With an incubation period of 5 days, the corresponding growth of infections reached its maximum on 5 March, before the first NPI became effective. Therefore, it is obvious that the 
spread of the virus was already in decline before the first intervention. And it was even negative before the extensive lockdown.

In a recent addendum [10], to their original article [5], Dehning et al. reconsider their model, using incident cases rather than reported cases. Their new principal result, shown in Fig 3, corroborates our finding that Germany's lockdown was superfluous.

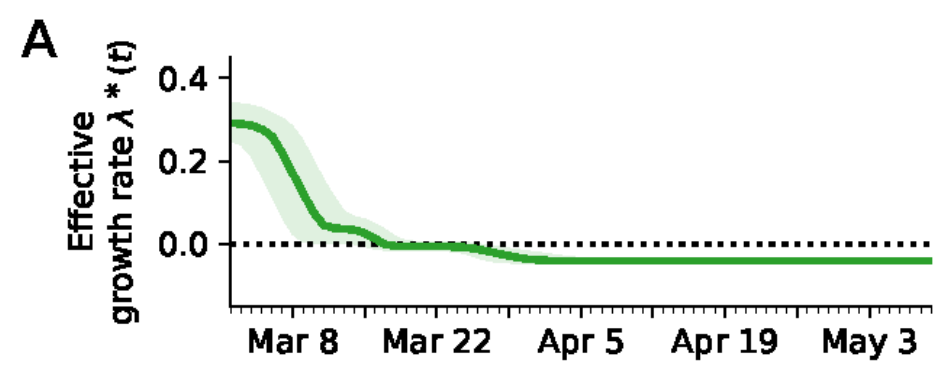

Fig 3. Effective growth rate of infections in Germany. Source: [10], Fig. 17A.

Fig 3 documents that the effective growth rate of SARS-Cov-2 started declining sharply on March 7, i.e., before the first intervention. What is more, the effective growth rate became negative on March 17, six days before the lockdown. This finding, derived from the same model but using more reliable data, puts the original inference of Dehning et al. upside down: Germany's extensive lockdown and contact bans, effective from 23 March, were by no means necessary to stop the spread of the virus.

\section{Conclusion}

In evaluating the necessity of NPIs during the corona crises, data quality is crucially important. Employing reported cases yields meaningless results because these data come with uncertain and variable lags that make it impossible to determine the path of the spread of the 
virus precisely. Using incident cases is highly preferable if such data are available. With known dates of the onset of symptoms, researches must only subtract an incubation period of 5 days to determine the true dynamics of infections.

Official data from Germany's RKI agency suggest strongly that the spread of the corona virus in Germany receded autonomously, before any interventions become effective. Several reasons for such an autonomous decline have been suggested. One is that differences in host susceptibility and behavior can result in herd immunity at a relatively low prevalence level. Accounting for individual variation in susceptibility or exposure to the coronavirus yields a maximum of $17 \%$ to $20 \%$ of the population that needs to be infected to reach herd immunity [11], an estimate that is empirically supported by the cohort of the Diamond Princess cruise ship [12]. Another reason is that seasonality may also play an important role in dissipation [13].

Finally, the ineffectiveness of the NPIs is also supported by empirical studies that determine their effects directly: Recent studies have shown that children are less involved in the transmission of SARS-CoV-2 than adults [14,15], suggesting that the closure of schools and kindergartens contributes little to curbing the spread of SARS-CoV-2. This finding is supported by studies of previous pandemics: A review of the effects of school closures regarding the spread of SARS-1 in China, Hong Kong, and Singapore in 2003 found only marginal effects [16]. A more recent study that employs German county data, [17], is also fully consistent with our findings.

\section{References}

1. Keogh-Brown MR, Smith RD, Edmunds JW, Beutels P. The macroeconomic impact of pandemic influenza: estimates from models of the United Kingdom, France, Belgium and the Netherlands. Eur J Health Econ 2010; 11: 543-554. 
2. Brooks SK, Webster RK, Smith LE, Woodland L, Wessely S, Greenberg N, et al. The psychological impact of quarantine and how to reduce it: rapid review of the evidence. Lancet 2020; 395: 912-920.

3. Galea S, Merchant RM, Lurie N. The Mental Health Consequences of COVID-19 and Physical Distancing: The Need for Prevention and Early Intervention. JAMA 2020; Forthcoming.

4. CovidSurg Collaborative, Nepogodiev D, Bhangu A. Elective surgery cancellations due to the COVID-19 pandemic: global predictive modelling to inform surgical recovery plans. Br J Surg 2020; Forthcoming.

5. Dehning J, Zierenberg J, Spitzner FP, Wibral M, Neto JP, Wilczek M, et al. Inferring change points in the spread of COVID-19 reveals the effectiveness of interventions. Science 2020; Forthcoming.

6. Robert Koch Institute. Answers to frequently asked questions about the SARS-CoV-2 coronavirus: Why are the data on COVID 19 cases reported by RKI and Johns Hopkins University in Germany different? [in German]. Available from: https://www.rki.de/SharedDocs/FAQ/ NCOV2019/gesamt.html. Accessed 21 May 2020.

7. Buchholz U, Buda S, Prahm KU. Abrupter Rückgang der Raten an Atemwegserkrankungen in der deutschen Bevölkerung [Abrupt decline in the rates of respiratory diseases in the German population]. Epid Bull 2020; 16: 7-9.

8. an der Heiden M, Hamouda O. Schätzung der aktuellen Entwicklung der SARS-CoV-2Epidemie in Deutschland - Nowcasting. [Estimation of actual development of the SARSCoV2 epidemics in Germany]. Epid Bull 2020; 17: 10-15. 
9. Lauer SA, Grantz KH, Bi Q, Jones FK, Zheng Q, Meredith HR, et al. The Incubation Period of Coronavirus Disease 2019 (COVID-19) From Publicly Reported Confirmed Cases: Estimation and Application. Ann Int Med 2020; Forthcoming.

10. Dehning J, Spitzner P, Linden M, Mohr SB, Neto JP, Zierenberg J, et al. Model-based and model-free characterization of epidemic outbreaks - Technical notes on Dehning et al., Science, 2020. Available from: https://github.com/PriesemannGroup/covid19_inference_forecast/blob/master/technical_notes.md (2020b). Accessed 17 Juni 2020.

11. Gomes MGM, Corder RM, King JG, Langwig KE, Souto-Maior C, Carneiro J, et al. Individual variation in susceptibility or exposure to SARS-CoV-2 lowers the herd immunity threshold. medRxiv 2020.04.27.20081893 [Preprint]. Available from: https://www.medrxiv.org/content/10.1101/2020.04.27.20081893v3

12. Russell TW, Hellewell J, Jarvis CI, van Zandvoort K, Abbott S, Ratnayake R, et al., Estimating the infection and case fatality ratio for COVID-19 using age-adjusted data from the outbreak on the Diamond Princess cruise ship. Eurosurveillance 2020; 25:2000256.

13. Evangelista AT. The Seasonal End of Human Coronavirus Hospital Admissions with Implications for SARS-CoV-2. medRxiv 2020.05.15.20103416 [Preprint]. Available from: https://www.medrxiv.org/content/10.1101/2020.05.15.20103416v1

14. Davies NG, Klepac P, Liu Y, Prem K, Jit M, CMMID COVID-19 working group, et al. Agedependent effects in the transmission and control of COVID-19 epidemics. medRxiv 2020.03.24.20043018 [Preprint]. Available from: https://www.medrxiv.org/content/10.1101/2020.03.24.20043018v2. 
15. Danis K, Epaulard O, Bénet T, Gaymard A, Campoy S, Botelho-Nevers E, et al. Cluster of coronavirus disease 2019 (Covid-19) in the French Alps. Clin Infect Dis 2020. Fortcoming.

16. Viner RM, Russell SJ, Croker H, Packer J, Ward J, Stansfield C, et al. School closure and management practices during coronavirus outbreaks including COVID-19: a rapid systematic review. Lancet Child Adolesc Health 2020; 4: 397-404.

17. Wieland T. Flatten the Curve! Modeling SARS-CoV-2/COVID-19 Growth in Germany on the County Level [Preprint]. Available from: https://www.researchgate.net/publication/341376926_Flatten_the_Curve_Modeling_SARSCoV-2COVID-19_Growth_in_Germany_on_the_County_Level. Accessed 5 June 2020.

Acknowledgments: The authors declare that they have no conflicts of interest. 